\title{
Large area metasurface lenses in the NIR region
}

\section{Mitchell Kenney, James Grant, Danni Hao, Kevin Docherty, Gordon Mills, et al.}

Mitchell Kenney, James Grant, Danni Hao, Kevin Docherty, Gordon Mills, Graham Jeffrey, Donald Macleod, David Henry, Peter MacKay, Marc Sorel, Robert A. Lamb, David R. S. Cumming, "Large area metasurface lenses in the NIR region," Proc. SPIE 11057, Modeling Aspects in Optical Metrology VII, 110570C (21 June 2019); doi: 10.1117/12.2527157

SPIE. Event: SPIE Optical Metrology, 2019, Munich, Germany 


\title{
Large area metasurface lenses in the NIR region
}

\author{
Mitchell Kenney*a, James Grant ${ }^{\mathrm{a}}$, Danni Hao ${ }^{\mathrm{a}}$, Kevin Docherty ${ }^{\mathrm{b}}$, Gordon Mills ${ }^{\mathrm{b}}$, Graham Jeffrey ${ }^{\mathrm{c}}$, \\ Donald Macleod ${ }^{\mathrm{d}}$, David Henry ${ }^{\mathrm{d}}$, Peter MacKay ${ }^{\mathrm{e}}$, Marc Sorel ${ }^{\mathrm{a}}$, Robert A. Lamb ${ }^{\mathrm{c}}$, \\ David. R. S. Cumming ${ }^{\mathrm{a}}$

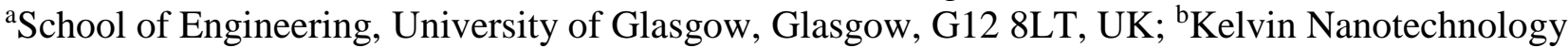 \\ Ltd., 70 Oakfield Avenue, Glasgow, G12 8LS, UK; 'Leonardo MW Ltd., 2 Crewe Road North, \\ Edinburgh, EH5 2XS, UK; ${ }^{d}$ STFC UK Astronomy Technology Centre, Royal Observatory \\ Edinburgh, Blackford Hill, Edinburgh, EH9 3HJ, UK; ${ }^{\mathrm{e}}$ Gooch \& Housego PLC, Dowlish Ford, \\ Ilminster, TA19 0PF, UK
}

\begin{abstract}
Metasurfaces have revolutionized the definition of compact optics. Using subwavelength periodic structures of nanostructured dielectrics, the refractive index and absorption properties of metasurfaces - which are 2D metamaterials can manipulate light to a degree not possible with conventional bulk glasses and crystals. The phase, polarization, spin (for circularly polarized light), amplitude and wavelength of light can all be manipulated and crafted to user-specified values to mimic the action of a lens, which we refer to as a metalens (ML). MLs have four major advantages over traditional refractive lenses - superior resolution, lighter weight, miniaturization and cost. Many metasurfaces with useful functionalities have been proposed in recent years, yet although novel in their approach have few real-world applications. One such market is the use within infrared laser systems, such as laser designators. In this work, we demonstrate metasurface lenses working at a wavelength of $\lambda=1064 \mathrm{~nm}$, with aperture $\mathrm{d}=1 \mathrm{~mm}$ and four different $\mathrm{F}$ numbers (focal length $\mathrm{f}=0.5,1,2$ and $5 \mathrm{~mm}$ ). The lenses are composed of 700nm high a-Si pillars - ranging from 70$360 \mathrm{~nm}$ diameter - which are fabricated using electron beam lithography (EBL) and reactive ion etching processes, on top of a fused silica substrate. Such lenses are shown to have diffraction-limited performance, with focal spot-size agreeing with theoretical values of $\lambda \cdot \mathrm{f} / \mathrm{d}$. Furthermore, we have designed large area lenses with aperture $\mathrm{d}=10 \mathrm{~mm}$, where the number of pillars per lens exceeds 550 million. By using an efficient Python script, we are able to produce these $100 \mathrm{~mm}^{2}$ samples with just 14 hours of EBL writing time.
\end{abstract}

Keywords: Metalens, metasurface, dielectric, metamaterial, ultra-thin lens, nanostructure, infrared, large-area

\section{INTRODUCTION}

Metasurfaces - 2D metamaterials - have changed the way we imagine compact optics, and set a new threshold for miniaturization. Previous approaches to produce miniature optical components have involved gradient-index (GRIN) materials, such as for GRIN lenses, as well as typical Fresnel-type lenses, and binary gratings. These are arguably still relatively bulky, when compared with the wavelength of the light they are designed for, and rely on classical optical phenomena, i.e. refractive optics (RO), involving the bulk refractive index to shape the wavefront of the light. Metasurfaces, however, are true miniature optical components, where the thickness is typically less than one wavelength of light (subwavelength). These metasurfaces are typically an area composed of periodic scatterers (either metallic or dielectric), which are themselves subwavelength in the in-plane direction. Indeed, it is necessary for metasurfaces to have their periodicity less than the wavelength of interest to avoid diffraction effects. The scatterers are able to control aspects of the wavefront of incident, such as polarization, phase, amplitude, and transmission-reflection ratio as a means to arbitrarily shape the wavefront of light as we see fit. This has resulted in some remarkable devices, that can achieve previously unheard of phenomena and modes of operation ${ }^{[1-12]}$.

In recent years, extensive focus has been on the development of lensing metasurfaces, namely metalenses or ultrathin

*Mitchell.Kenney@glasgow.ac.uk; phone +44 1413306690

Modeling Aspects in Optical Metrology VII, edited by Bernd Bodermann, Karsten Frenner, Proc. of SPIE Vol. 11057, 110570C · (C) 2019 SPIE · CCC code: 0277-786X/19/\$21 · doi: 10.1117/12.2527157 
lenses (UTL's) [REF]. Early renditions of these utilized the benefit of plasmonic resonance along with the well-known Pancharatnam-Berry phase (Geometric phase) ${ }^{[13-16]}$. An issue with these lenses though is the very low efficiency of less than $15 \%$ - inherent to plasmonic devices in general - along with the small lens areas in the visible to Near Infrared (NIR) regimes. The plasmonic structures, which often also rely on the use of Au (gold), also prevent these lenses from operating below $\sim 550 \mathrm{~nm}$ wavelength and so could not be used to function across the whole visible spectrum. Addressing the wavelength issue can be achieved through the selection of other plasmonic metals, such as Ag (silver) or $\mathrm{Al}$ (aluminum), yet the problem of low efficiency still remains.

Following on from this work, an emergence of dielectric based metasurfaces devices started to be investigated. These dielectric metasurfaces are composed of nano-pillars, -posts, or -fins, where the dielectric materials used typically have relatively high refractive indices. These nanostructures inherently behave like truncated waveguides which support multimodal resonances, and produce a locally varying phase dependent upon the width of the posts. Because these dielectric are essentially lossless and behalf as waveguides, the efficiency of the transmitted light can be extremely high, and capable of exceeding $90 \%$ efficiency. The height of these nanopillars are much greater than those used in plasmonic metasurface devices, yet are still subwavelength. Owing to these dielectric nanopillars, many highly-efficient metalens devices have been investigated and published in the visible up to mid infrared $\left[^{5,7,17-27}\right]$. Recent works have improved upon these dielectric lenses, which were originally monochromatic by design, to allow them to operate over a brad bandwidth (achromatic operation) by modifying the nanopillar designs to include group-phase delays - the group-phase allowed the dispersion-dependent focal length changes to be negated with varying wavelength $\left.{ }^{28-32}\right]$. These seminal works have undoubtedly changed the landscape for what can be achieved with dielectric metalenses, yet the issue of large areas still remains a difficult feat of engineering to master, where only a few works have tried to address this $\left.{ }^{33-35}\right]$. The issue lies in the fact that the number of nanostructures required to produce lenses scales with the area, and so to produce a lens at $10 \mathrm{~mm}$ aperture would require 100x more nanostructures than a $1 \mathrm{~mm}$ lens. The number of elements is then approaching billions rather than millions, and computational power becomes a key consideration as file sizes also scale with the lens size. It is not then uncommon for pattern file sizes to be many gigabytes. This then passes forward onto the fabrication equipment, where some EBL tools (typically used to fabricate the small nanostructures in metalenses) simply cannot deal with such enormous data.

In this paper, we report large-area Ultrathin Lenses (UTLs), which we refer to as metalenses, measuring $1 \mathrm{~mm} \times 1 \mathrm{~mm}$ and are composed of in excess of 8 million periodic nanostructures of differing geometries. Four lenses with an operating wavelength of $1064 \mathrm{~nm}$ are designed, with different focal lengths of $0.5,1,2$ and $5 \mathrm{~mm}$, respectively - thereby giving us lenses of differing F-numbers. The lenses are fabricated using EBL and dry etching of amorphous silicon to produce circular nanopillars of $700 \mathrm{~nm}$ height, with diameters ranging between $80-330 \mathrm{~nm}$ to vary the phase. The lenses are shown to achieve near diffraction-limited performance, and able to resolve features down to $8.8 \mu \mathrm{m}$ (as determined by a USAF 1951 target). Additionally, the focussing efficiency is measured as being up to $67 \%$ with respect to the fused silica substrate. Finally, as a proof-of-concept we fabricate $10 \mathrm{~mm}$ aperture lenses with focal lengths of $2 \mathrm{~cm}$, which consist of nearly 1 billion nanopillars, yet only requires $\sim 15$ hours EBL writing time per lens. These lenses are

\section{DESIGN OF DIELECTRIC METALENSES}

\subsection{Numerical simulations in CST Microwave Studio}

A metalens, composed of meta-atoms, requires a varying phase profile across its area. These meta-atoms need to have the design capability of varying phase, which can be achieved by the use of dielectric cylindrical nanopillars. The phase shifting is achieved by varying the diameter of these nanopillars from a minimum (for 0 rad phase) to a maximum (for $2 \pi$ rad phase) value. To achieve this, we chose amorphous silicon as the material to construct the nanopillars, due to its relatively high refractive index $(\mathrm{n}=3.40)$ and low losses in the NIR region $(1064 \mathrm{~nm}$ wavelength). To assure high efficiency and reduction of scattering, we chose a triangular lattice and periodicity of $450 \mathrm{~nm}$, with a pillar height of 720 $\mathrm{nm}$. The widths of these pillars were swept from $60 \mathrm{~nm}$ to $350 \mathrm{~nm}$, as shown in Figure 1. We see in Figure 1c that the phase coverage from 0 to $2 \pi$ can be achieved with a pillar range of $60 \mathrm{~nm}$ up to $330 \mathrm{~nm}$, whilst maintaining a highefficiency above $80 \%$ regardless of pillar diameter. A sharp dip can be seen at $\sim 300 \mathrm{~nm}$, which we attribute to an anti- 
reflection or Fabry Perot type mode within the nanopillars. Because of this, we are sure not to choose pillars around this value so as to reduce any spurious effects in our lens design.

(a)

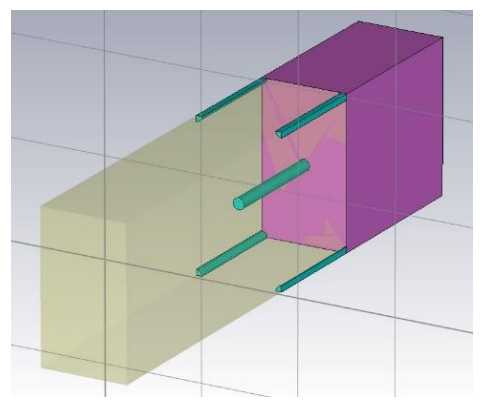

(b)

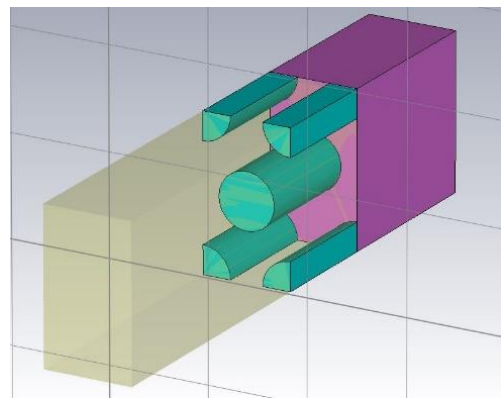

(c)

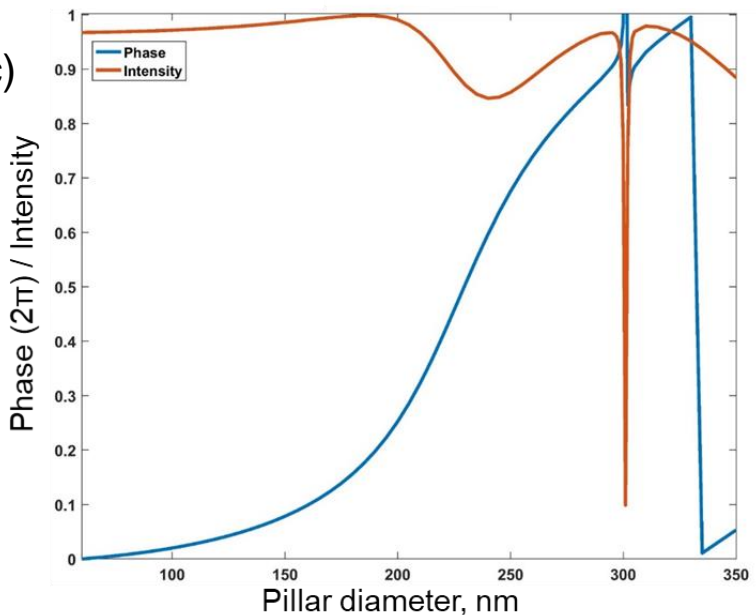

Figure 1. Schematic views of the nanopillars simulated in CST Microwave studio, for both the (a) minimum pillar size $(60 \mathrm{~nm})$ and $(b)$ maximum pillar size $(330 \mathrm{~nm})$. The material used for the nanopillars is amorphous silicon (teal colour) with a refractive index value of 3.40. The substrate (fused silica, purple) has a refractive index of 1.45 . Unit cell periodicity is

\subsection{Metalens pattern design and generation}

If we consider a general lens with a quadratically varying phase profile across a surface, with a focal length $f$ and wavelength of operation $\lambda$, we can use the following equation to govern the individual phase elements required at specific locations across a surface:

$$
\varphi=\frac{2 \pi}{\lambda}\left(\sqrt{f^{2}+x^{2}+y^{2}}-f\right)
$$

where $x, y$ are the coordinates of the nanopillars, and $\varphi$ is the phase at this location. Once we know the phase values required at each $(x, y)$ location to produce a lens we can directly associate each location with a nanopillar of a specific diameter from Figure 1c. 
With this information it is then possible to generate a lens pattern, via software coding. In our case we opted to use Matlab, and because it is not feasible to fabricate nanopillars with widths varying by less than $\sim 10 \mathrm{~nm}$, we opted to choose 25 nanopillar "bucket" values increasing by $10 \mathrm{~nm}$. In this, we are also sure to avoid the $300 \mathrm{~nm}$ diameter nanopillars due to the aforementioned anti-reflection response. From Matlab, we are then able to export txt. data files containing the patterns necessary to fabricate a metalens of a specific aperture, focal length, and operation wavelength in terms of nanopillar diameter and location across a surface.

\section{FABRICATION OF 1 MM METALENSES}

\subsection{Dose test trials for uniform pillar arrays}

A necessary step is to first investigate the feasible nanopillar diameters which fabrication allows. Firstly, amorphous silicon (a-Si) of $720 \mathrm{~nm}$ thickness was deposited onto fused silica substrates (500 $\mu \mathrm{m}$ thick). Following this, a bilayer PMMA was spin-coated onto the a-Si and a thin $20 \mathrm{~nm}$ aluminium conducting layer was deposited onto the PMMA to prevent charging when performing EBL. To do the EBL writing, a modified Vistec VB6 EBL tool was used with a 100 $\mathrm{kV}$ beam. Different doses were trialled for the 25 differing nanopillar widths and a lookup table produced. This allowed us to arbitrarily choose a specific dose current to achieve the nanopillar diameters chosen from the CST simulations. Following EBL, the charge conduction layer was removed and PMMA resist developed to leave "voids". Next, a $50 \mathrm{~nm}$ $\mathrm{NiCr}$ layer was deposited to act as an etching hard mask, and standard metal lift-off process performed to leave behind $\mathrm{NiCr}$ disks. Reactive Ion Etching (RIE) using a C4F8/SF6 recipe was then carried out to etch the amorphous silicon, where the $\mathrm{NiCr}$ discs protect the pillar beneath. Finally, a UV exposure was carried out to break down the fluoropolymer layer formed during etching, and $\mathrm{NiCr}$ mask removed to leave behind the free-standing a-Si nanopillar arrays. Examples of these can be seen below in Figure 2.
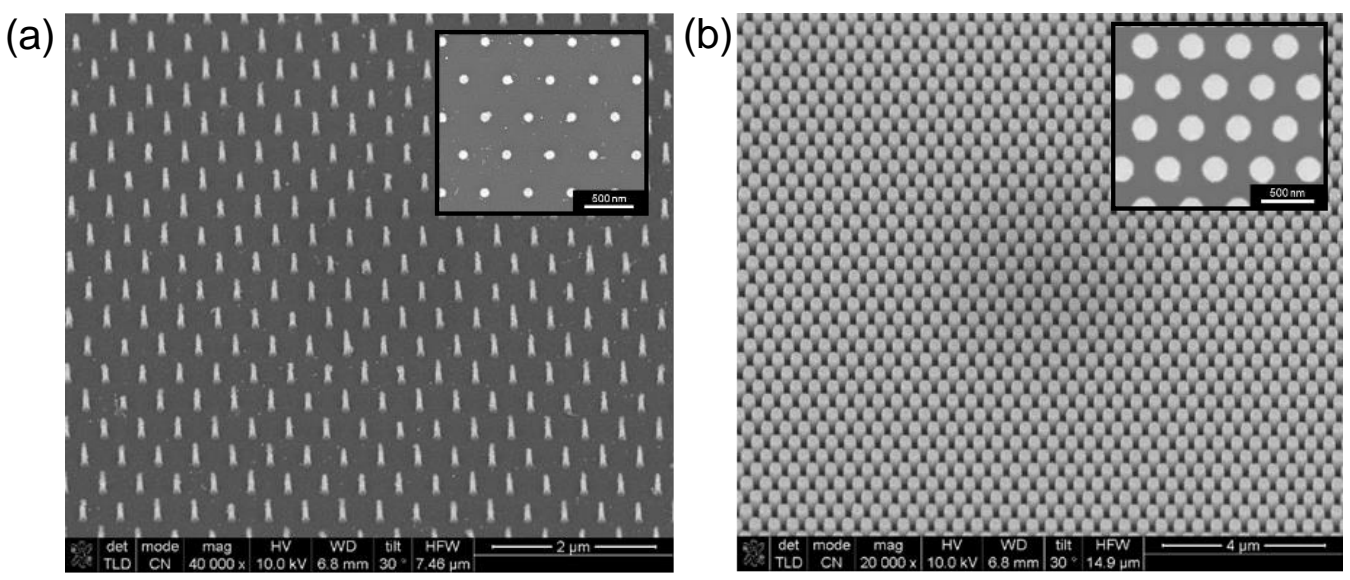

Figure 2. Scanning Electron Micrographs of etched a-Si nanopillars, with periodicity of $450 \mathrm{~nm}$. (a) Nanopillar arrays with diameter $80 \mathrm{~nm}$, imaged at an angle of $30^{\circ}$. Scale bar $-2 \mu \mathrm{m}$; Inset - the same nanopillars imaged top-down, scale bar $500 \mathrm{~nm}$. (b) Nanopillar arrays with diameter $330 \mathrm{~nm}$, imaged at an angle of $30^{\circ}$. Scale bar $-4 \mu \mathrm{m}$; Inset - the same nanopillars imaged top-down, scale bar $500 \mathrm{~nm}$.

\subsection{Fabrication of $1 \mathrm{~mm}$ metalenses}

After performing dose test trials to produce free-standing arrays of nanopillars, we then proceeded to generate the pattern files for the $1 \mathrm{~mm}$ lenses using the data files exported from both Matlab, CST and the lookup table from the dose tests. Four pattern files were generated corresponding to four lenses with different focal lengths (F-numbers, F\#'s). File sizes exported from Matlab were $\sim 300 \mathrm{MB}$ and took $\sim 2$ hours to generate. Industrial partners at Kelvin Nanotechnology 
(KNT) then used a bespoke Python code to convert the large data files in compressed, hierarchical files in WRVEP format (readable by the VB6), which is a much more efficient process for performing EBL with large file sizes. Additionally, the doses for all lenses was increased by $+10 \%$ to investigate the effect on fabrication tolerances and characterization comparisons for error analysis. This was doubled to produce 16 lenses, which are shown below in Figure 3a. Zoomed in electron microscope images o the F\#0.5 lenses (normal dose) are shown in Figure 3b and 3c.

(a)

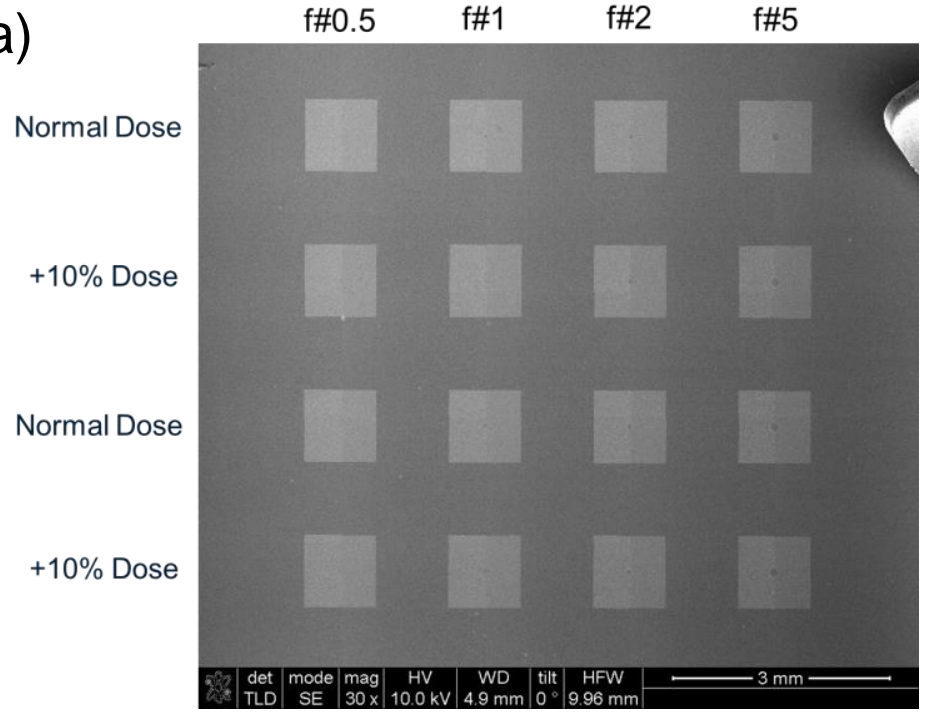

(b)
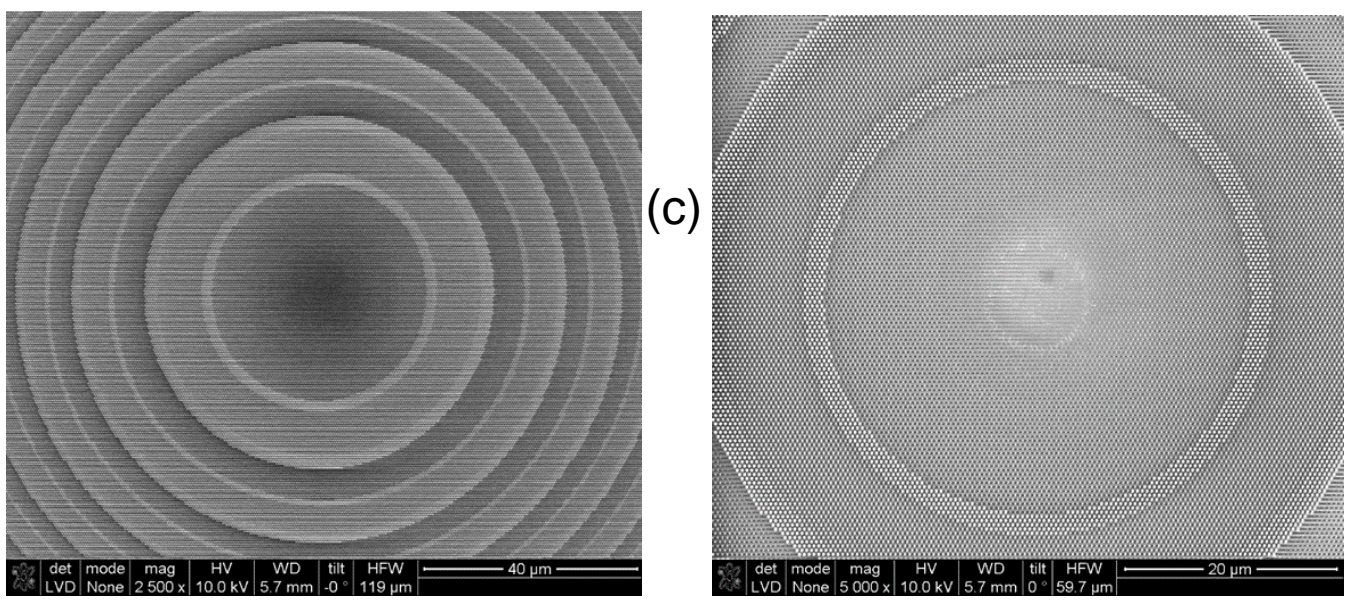

Figure 3. Scanning Electron Micrographs. (a) Sixteen metalens sample designs, with four differing focal lengths and two differing EBL doses. (b) $1 \mathrm{~mm}$ aperture metalens with focal length $0.5 \mathrm{~mm}$ (F\# 0.5) viewed at a magnification of 2,500x (Scale bar $-40 \mu \mathrm{m})$. (b) The same $1 \mathrm{~mm}$ aperture metalens viewed at a magnification of 5,000x (Scale bar $-20 \mu \mathrm{m}$ ).

\section{EXPERIMENTAL CHARACTERIZATION}

\subsection{Lens focussing characterization}

A bespoke optical setup was constructed to characterize the $1 \mathrm{~mm}$ metalenses, comprising of a NIR camera (Hamamatsu C10633), Zeiss Epiplan 50x objective lens, and an NKT Supercontinuum laser. The setup is shown below in Figure 4. 
The NKT Supercontinuum laser allows a broadband source of light, and so we inserted a $1064 \mathrm{~nm}$ filter (10nm linewidth) and neutral density (ND1) filter to prevent damage to the sensitive camera. Alignment was carried out by switching out the $1064 \mathrm{~nm}$ filter for a $633 \mathrm{~nm}$ filter, for ease of viewing. The metalenses were fixed to a multidirectional XYZ stage, allowing freedom of movement for choosing a specific metalens. The camera and objective lens were affixed to a breadboard and moved as one unit for investigating the focusing characteristics of the lenses.

(a)

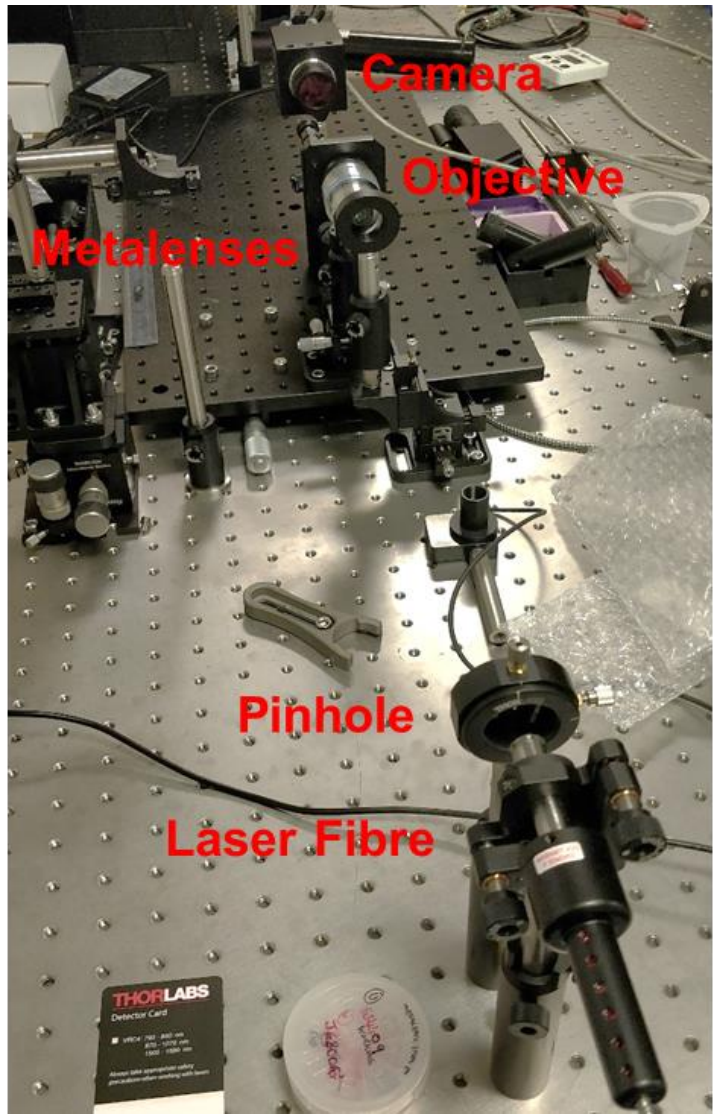

(b)

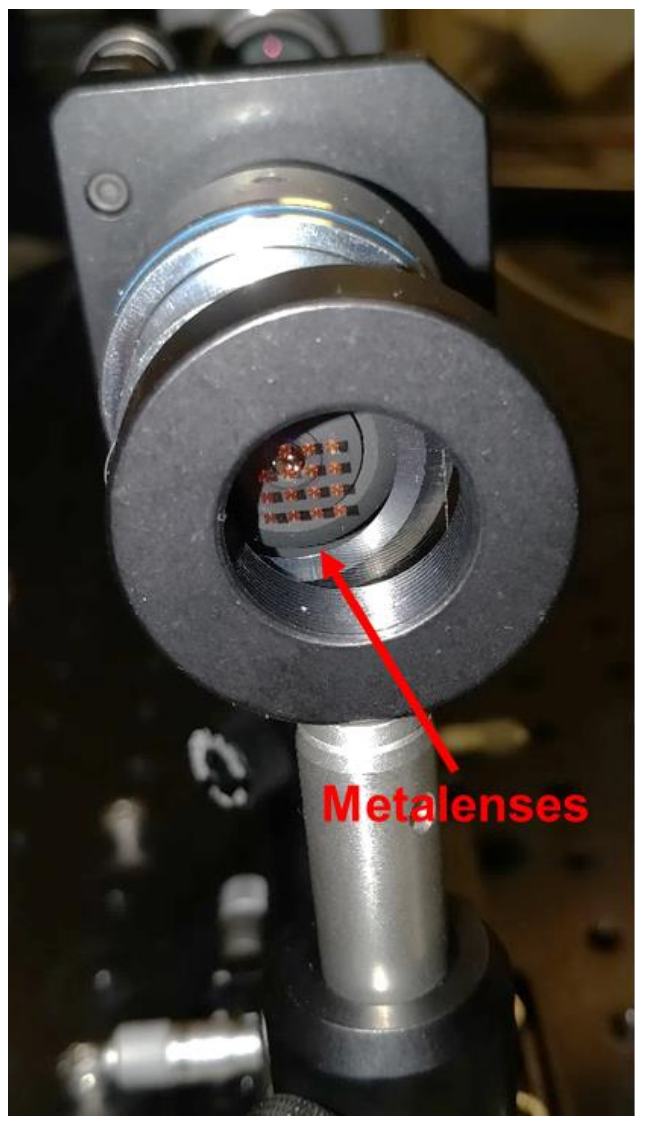

Figure 4. (a) Experimental characterization setup used to characterize and measure the focusing performances of the metalenses. (b) Close-up image showing the sample substrate with the sixteen differing metalenses on, positioned in front of the objective lens.

Images obtained from the camera are shown below in Figure 5. Figures 5a-d correspond to the focal lengths of $5 \mathrm{~mm}$ to $0.5 \mathrm{~mm}$, respectively. The focussing performance of a lens can be characterised by its resolution, where the spot size (SS) - namely the diffraction limit - can be calculated using the wavelength of interest, its focal length, and aperture size as given by the $\mathrm{SS}=\lambda \cdot f / d$. Using this equation, we calculate the (theoretical) diffraction limited spot size of the $5 \mathrm{~mm}$ lens (Figure 5a) as being $5.32 \mu \mathrm{m}$. The Hamamatsu NIR camera has sensing pixel sizes of $30 \times 30 \mu \mathrm{m}$, and therefore we can deduce the physical spot size using this information. As measured in Figure 5a, the central focal spot measures 17 pixels across, corresponding to a physical spot size of $510 \mu \mathrm{m}$. Dividing this value by 50 (because of the $50 \mathrm{x}$ magnification from the objective), we obtain a physical spot value of $\sim 10.2 \mu \mathrm{m}$. Assuming a Gaussian beam profile, the FWHM value of this spot is then $\sim 5.1 \mu \mathrm{m}$ - this value is very close to the theoretically calculated value of $5.32 \mu \mathrm{m}$, and therefore our metalenses are near diffraction-limited and should be able to resolve fine features. Because the spot size is a function of its focal length, we can infer that smaller focal length lenses will produce smaller spot sizes, and therefore be able to resolve finer details. However, the depth of filed also increases, which makes imaging over small distances in the beam direction more difficult, especially true for $1 \mathrm{~mm}$ lenses and smaller. 

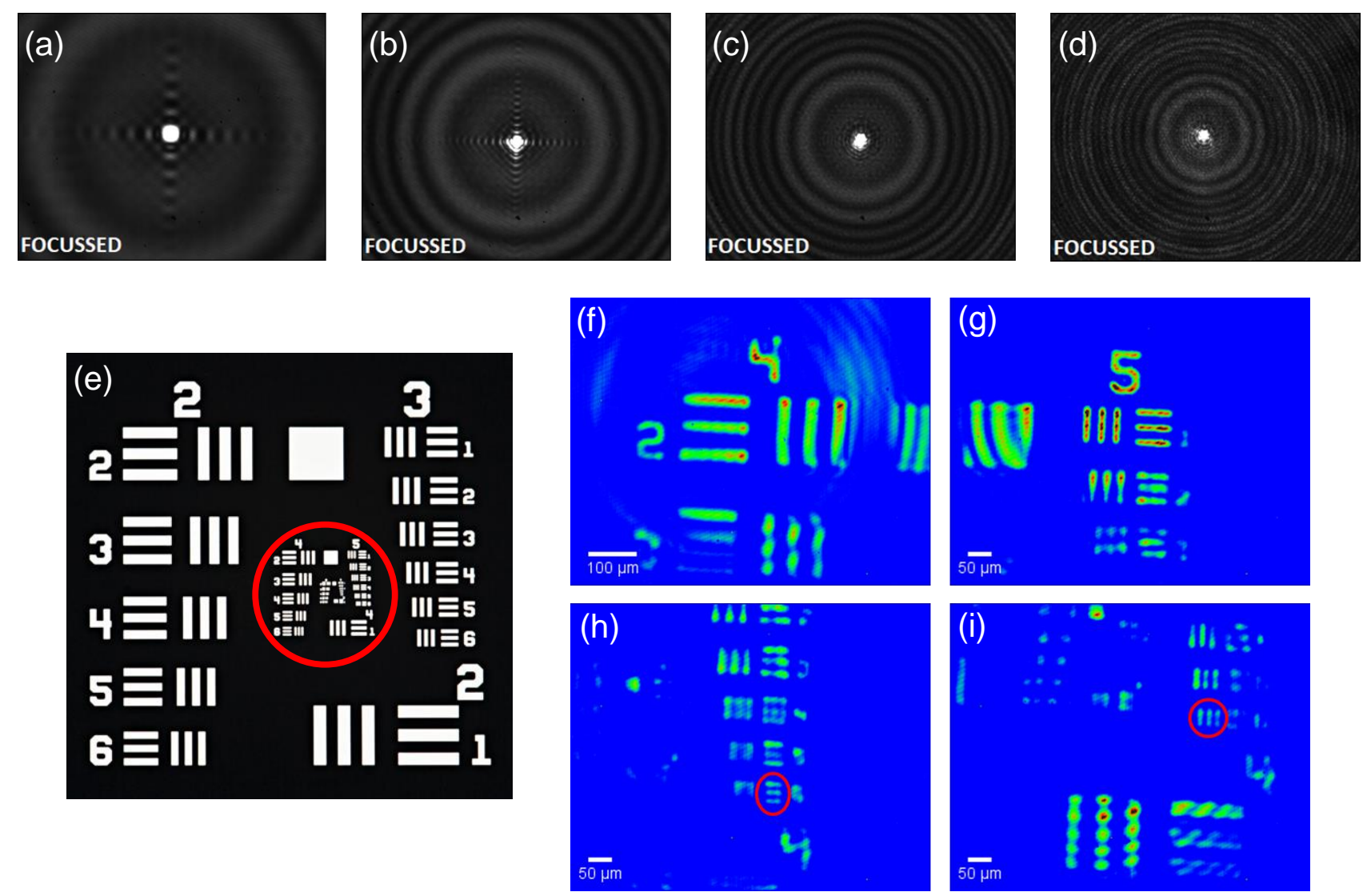

Figure 5 (a) Focussed beam spot for the F\#5 metalens. (b) Focussed beam spot for the F\#2 metalens. (c) Focussed beam spot for the F\#1 metalens. (d) Focussed beam spot for the F\#0.5 metalens. All images with a 50x objective lens. (e) Commercial US AirForce (USAF) 1951 target, used for imaging resolution measurements. The red circle highlights the region which was imaged. (f) Top-left of the imaged region. (g) Top-right of the imaged region. (h) Mid-right of the imaged region. The red circle illustrates the minimum resolvable feature, located at Group 5 element 6 which corresponds to a feature size of $8.8 \mu \mathrm{m}$ (17.6 $\mu \mathrm{m}$ linepair size). (h) Bottom-right of the imaged region. The red circle illustrates the same minimum resolvable feature as in (h), which is $8.8 \mu \mathrm{m}$, except for the vertical patterns.

\subsection{Imaging performance using a USAF 1951 resolution target}

After characterizing the metalenses, the next stage was to assess their performance in an imaging setup. The optical setup shown in Figure 4 was slightly modified so that a resolvable object was placed before the metalenses. We chose to use a USAF 1951 resolution target due to its unparalleled and widespread use in determining the imaging performance and resolution capabilities of optical systems. A schematic of the USAF 1951 target is shown in Figure 5e, where the red circle highlights the imaging region of interest which approaches the resolving capability of our metalenses. Using our metalens with focal length $5 \mathrm{~mm}$, we obtained a number of images relating to different areas of the pattern within the red circle in Figure 5e, and these are shown in Figure 5f-5i. Clear features can be resolved, with numbers and triplet linepairs being clear in all four sub-figures. The minimum resolvable features in which our F\#5 metalens could image are given in the red circles in Figure 5h and Figure 5i, which correspond to Group 5 element 6. From the typical data sheet, this pattern corresponds to a linewidth of $8.8 \mu \mathrm{m}$ (17.6 $\mu \mathrm{m}$ linepair width). This value is very close to our measured spot size of $5.1 \mu \mathrm{m}$ and therefore we can conclude that the metalenses can be used for near diffraction limited imaging performance. 


\section{PROOF-OF-CONCEPT LARGE-AREA METALENSES}

\subsection{Fabrication of $10 \mathrm{~mm}$ aperture metalenses}

As a proof of concept to demonstrate the feasibility of upscaling $1 \mathrm{~mm}$ metalenses, we repeated the previous methods to develop a $10 \mathrm{~mm}$ lens. Many steps remained unchanged, such as the resist spin-coating, development, metallization, and dry etching. However, some of the previous steps had to be addressed to address the size increase of the metalens namely the code generation and EBL process. Whilst it is relatively straightforward and not significantly taxing to fabricate $1 \mathrm{~mm}$ metalenses, the $10 \mathrm{~mm}$ metalenses had many issues which had to be taken into consideration. The generated pattern files for the $1 \mathrm{~mm}$ lenses were only $300 \mathrm{MB}$ in size and required just a few hours to generate - the file size after Python conversion was also easily handled by the VB6 EBL tool. However, upscaling to $10 \mathrm{~mm}$ metalenses requires a 100-fold in the written area, and therefore requires that 100x more nanostructures in the pattern file be generated. This results in a file size of $\sim 30 \mathrm{~GB}$, which takes $\sim 2.5-3$ days to generate, and is also too large to be handled directly by the VB6 EBL tool (even after conversion with Python). To address this, the Python code was modified to split the $10 \times 10 \mathrm{~mm}$ patterned area into one hundred smaller $1 \times 1 \mathrm{~mm}$ areas and additionally to limit the data which was allowed to be accessed to partitions of < $500 \mathrm{MB}$; the VB6 EBL tool has the capability of utilizing its 2x 500 MB memory banks to handle two sets of data simultaneously such that there are minimal bottlenecks when reading data and writing the patterns.

Utilising this method, the VB6 EBL tool was able to write the $10 \mathrm{~mm}$ metalens in $\sim 15$ hours. Various images of the 10 $\mathrm{mm}$ metalens are shown in Figure 6, including photograph (Figure 6a), optical microscope (Figure 6b), and Electron microscope (Figure 6c). We position the metalens alongside a standard measuring ruler for size reference in Figure 6a. In both Figure $6 \mathrm{~b}$ and $6 \mathrm{c}$ we can clearly see the phase zones owing to the differing sizes of the nanopillars composing the metalens.
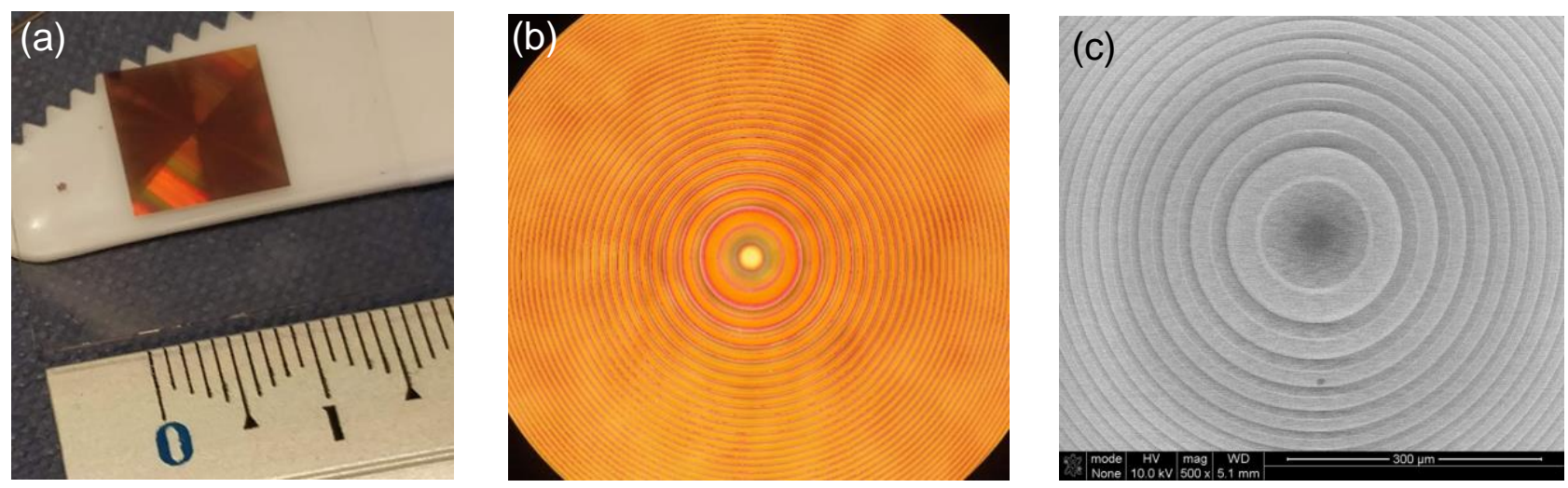

Figure 6. (a) Photograph of the fabricated $10 \mathrm{~mm}$ metalens sample, beside a standard measuring ruler for reference. (b) Optical micrograph of the $10 \mathrm{~mm}$ metalens sample, showing the zone features (50x mag). (c) Scanning Electron Micrograph of the etched $10 \mathrm{~mm}$ metalens, demonstrating the zones of differing pillar diameter. (scale bar: $300 \mu \mathrm{m}$ ).

Whilst $\sim 15$ hours may seem a long time to envision a viable pipeline for mass-production of these lenses, we believe that this time can be considerably reduced. For the first option, we would instead switch to a circular lens rather than a square; the extra space in the corners of the square lens is unused, and therefore removing these area would result in a $21 \%$ saving in the written area - which if applied to the writing time would mean a reduction of $\sim 3$ hours. Another improvement in the writing time may be achieved by approximating the circular nanostructures with a less fine edge granularity. The current method uses vertex sizes of the nanopillars as multiples of E-beam step size. Instead, we may choose a slightly larger beam step size with less smooth vertices composing the circular features. This would result in the E-beam having a smaller dwell time and can therefore write more features in a shorter time. We believe that using these extra time-saving methods could result in a 50\% reduction in the writing time of $10 \mathrm{~mm}$ metalenses, taking only around 7 hours. This then opens up the potential for increasing the metalens size even further, where a metalens of (for example) 
$20 \mathrm{~mm}$ aperture would only take $\sim 28$ hours to write. This opens up a whole host of exciting opportunities for a viable pipeline to produce large area multifunctional metasurface devices.

\section{CONCLUSION}

Commercial interest in metasurface devices has been hindered in previous years due to small sizes and low efficiencies of devices. Owing to the advent of dielectric metasurfaces with low losses, and world-leading fabrication techniques and equipment, large area and highly efficient metasurface devices are now of real value and potentially game-changing. A key interest lies in metasurface lenses, where typical bulky glass lenses may be replaced by these ultrathin, lightweight lenses and open up a range of new compact products. A particular interest is taken by the defense and space sectors, where the weight and number of optical components can directly be tied to the revenue lost due to weight tolerances. We have presented large area $(1-10 \mathrm{~mm})$ metalenses of differing focal lengths, capable of focusing with an efficiency of $67 \%$ and exhibiting near diffraction-limited performance, capable of resolving features as small as $9 \mu \mathrm{m}$ at a wavelength of $1064 \mathrm{~nm}$. Finally, we showcase $10 \mathrm{~mm}$ metalenses as a feasibility study which can be produced by EBL in just $\sim 15$ hours. We envision a reduction in the time to produce these lenses with some optimizations, so that such $10 \mathrm{~mm}$ lenses will only take 7-8 hours EBL writing time. Such work is of significant interest to the defense and space sectors, such as the Centre for Earth Observation Instrumentation (CEOI), where size and weight constraints mean thinner and lighter components are always at the forefront of emerging technologies.

\section{ACKNOWLEDGMENTS}

The authors would like to thank the staff of the James Watt Nanofabrication Centre at the University of Glasgow for assistance in fabricating the devices reported in this paper. Data sets used in this paper can be obtained from the following online repository <http://dx.doi.org/10.5525/gla.researchdata.838> or corresponding author. This work was supported by the Engineering and Physical Sciences Research Council of the United Kingdom, Grants No. EP/J018678/1 and EP/M01326X/1.

\section{REFERENCES}

[1] Yu, N., Genevet, P., Kats, M. a., Aieta, F., Tetienne, J.-P., Capasso, F. and Gaburro, Z., "Light propagation with phase discontinuities: generalized laws of reflection and refraction," Science 334(6054), 333-337 (2011).

[2] Yu, N. and Capasso, F., "Flat optics with designer metasurfaces," Nat. Mater. 13(2), 139-150 (2014).

[3] Kenney, M., Grant, J., Shah, Y. D., Escorcia-Carranza, I., Humphreys, M. and Cumming, D. R. S., "OctaveSpanning Broadband Absorption of Terahertz Light Using Metasurface Fractal-Cross Absorbers," ACS Photonics 4(10), 2604-2612 (2017).

[4] Shah, Y. D., Grant, J., Hao, D., Kenney, M., Pusino, V. and Cumming, D. R. S., "Ultra-narrow Line Width Polarization-Insensitive Filter Using a Symmetry-Breaking Selective Plasmonic Metasurface,” ACS Photonics 5(2) (2018).

[5] Arbabi, A., Horie, Y., Ball, A. J., Bagheri, M. and Faraon, A., "Subwavelength-thick lenses with high numerical apertures and large efficiency based on high-contrast transmitarrays," Nat. Commun. 6(1), 7069 (2015).

[6] Qin, L., Zhang, K., Peng, R. W., Xiong, X., Zhang, W., Huang, X. R. and Wang, M., "Optical-magnetisminduced transparency in a metamaterial,” Phys. Rev. B - Condens. Matter Mater. Phys. (2013).

[7] Khorasaninejad, M., Chen, W. T., Devlin, R. C., Oh, J., Zhu, A. Y. and Capasso, F., "Metalenses at visible wavelengths: Diffraction-limited focusing and subwavelength resolution imaging," Science (80-. ). 352(6290), 1190-1194 (2016).

[8] Huang, L., Chen, X., Mühlenbernd, H., Zhang, H., Chen, S., Bai, B., Tan, Q., Jin, G., Cheah, K.-W., Qiu, C.-W., Li, J., Zentgraf, T. and Zhang, S., "Three-dimensional optical holography using a plasmonic metasurface," Nat. Commun. 4(May), 2808. (2013).

[9] Zheng, G., Mühlenbernd, H., Kenney, M., Li, G., Zentgraf, T. and Zhang, S., "Metasurface holograms reaching 80\% efficiency," Nat. Nanotechnol. 10, 308-312 (2015).

[10] Fedotov, V. A., Schwanecke, A. S., Zheludev, N. I., Khardikov, V. V. and Prosvirnin, S. L., “Asymmetric 
Transmission of Light and Enantiomerically Sensitive Plasmon Resonance in Planar Chiral Nanostructures,” Nano Lett. 7(7), 1996-1999 (2007).

[11] Kenney, M., Li, S., Zhang, X., Su, X., Kim, T., Wang, D., Wu, D., Ouyang, C., Han, J., Zhang, W., Sun, H. and Zhang, S., "Pancharatnam-Berry Phase Induced Spin-Selective Transmission in Herringbone Dielectric Metamaterials," Adv. Mater. 28(43), 9567-9572 (2016).

[12] Liu, L., Zhang, X., Kenney, M., Su, X., Xu, N., Ouyang, C., Shi, Y., Han, J., Zhang, W. and Zhang, S., "Broadband metasurfaces with simultaneous control of phase and amplitude," Adv. Mater. 26(29), 5031-5036 (2014).

[13] Chen, X., Huang, L., Mühlenbernd, H., Li, G., Bai, B., Tan, Q., Jin, G., Qiu, C.-W., Zhang, S. and Zentgraf, T., "Dual-polarity plasmonic metalens for visible light," Nat. Commun. 3, 1198. (2012).

[14] Ni, X., Ishii, S., Kildishev, A. V and Shalaev, V. M., "Ultra-thin, planar, Babinet-inverted plasmonic metalenses," Light Sci. Appl. 2(4), e72. (2013).

[15] Aieta, F., Genevet, P., Kats, M. a., Yu, N., Blanchard, R., Gaburro, Z. and Capasso, F., “Aberration-Free Ultrathin Flat Lenses and Axicons at Telecom Wavelengths Based on Plasmonic Metasurfaces," Nano Lett. 12(9), 4932-4936 (2012).

[16] Chen, X., Huang, L., Mühlenbernd, H., Li, G., Bai, B., Tan, Q., Jin, G., Qiu, C.-W., Zentgraf, T. and Zhang, S., "Reversible Three-Dimensional Focusing of Visible Light with Ultrathin Plasmonic Flat Lens," Adv. Opt. Mater. 1(7), 517-521 (2013).

[17] Khorasaninejad, M. and Capasso, F., "Metalenses: Versatile multifunctional photonic components," Science (80. ). 358(6367) (2017).

[18] Chen, W. T., Zhu, A. Y., Khorasaninejad, M., Shi, Z., Sanjeev, V. and Capasso, F., "Immersion Meta-Lenses at Visible Wavelengths for Nanoscale Imaging," Nano Lett., acs.nanolett.7b00717 (2017).

[19] Capasso, F., "Review article The future and promise of flat optics : a personal perspective" (2018).

[20] Arbabi, A., Horie, Y., Bagheri, M. and Faraon, A., "Dielectric metasurfaces for complete control of phase and polarization with subwavelength spatial resolution and high transmission," Nat. Nanotechnol. 10(11), 937-943 (2015).

[21] Arbabi, A., Briggs, R. M., Horie, Y., Bagheri, M. and Faraon, A., "Efficient dielectric metasurface collimating lenses for mid-infrared quantum cascade lasers," Opt. Express 23(26), 33310 (2015).

[22] Arbabi, A., Arbabi, E., Kamali, S. M., Horie, Y., Han, S. and Faraon, A., "Miniature optical planar camera based on a wide-angle metasurface doublet corrected for monochromatic aberrations," Nat. Commun. 7, 1-9 (2016).

[23] Ile, Z. L. I., Heng, G. U. Z., Ing, P. A. N. H. E., Ong, S. L. I., Eng, Q. I. D., Hao, J. I. Z., Ong, Y. A. I., Li, Z., Zheng, G., He, P., Li, S., Deng, Q., Zhao, J. and Ai, Y., “All-silicon nanorod-based Dammann gratings," Opt. Lett. 40(18), 4285-4288 (2015).

[24] Zheng, G., Liu, G., Kenney, M. G., Li, Z., He, P., Li, S., Ren, Z. and Deng, Q., "Ultracompact high-efficiency polarising beam splitter based on silicon nanobrick arrays," Opt. Express 24(6), 6749 (2016).

[25] Khorasaninejad, M., Chen, W. T., Zhu, A. Y., Oh, J., Devlin, R. C., Rousso, D. and Capasso, F., "Multispectral Chiral Imaging with a Meta-lens," Nano Lett., acs.nanolett.6b01897 (2016).

[26] Zhu, A. Y., Chen, W.-T., Khorasaninejad, M., Oh, J., Zaidi, A., Mishra, I., Devlin, R. C. and Capasso, F., "Ultracompact visible chiral spectrometer with meta-lenses," APL Photonics 2(3), 036103 (2017).

[27] Khorasaninejad, M., Chen, W. T., Zhu, A. Y., Oh, J., Devlin, R. C., Roques-Carmes, C., Mishra, I. and Capasso, F., "Visible Wavelength Planar Metalenses Based on Titanium Dioxide," IEEE J. Sel. Top. Quantum Electron. 23(3) (2017).

[28] Wang, S., Wu, P. C., Su, V.-C., Lai, Y.-C., Hung Chu, C., Chen, J.-W., Lu, S.-H., Chen, J., Xu, B., Kuan, C.-H., Li, T., Zhu, S. and Tsai, D. P., "Broadband achromatic optical metasurface devices," Nat. Commun. 8(1), 187 (2017).

[29] Wang, S., Wu, P. C., Su, V.-C., Lai, Y.-C., Chen, M.-K., Kuo, H. Y., Chen, B. H., Chen, Y. H., Huang, T.-T., Wang, J.-H., Lin, R.-M., Kuan, C.-H., Li, T., Wang, Z., Zhu, S. and Tsai, D. P., “A broadband achromatic metalens in the visible," Nat. Nanotechnol. 13(3), 227-232 (2018).

[30] Shrestha, S., Overvig, A. C., Lu, M., Stein, A. and Yu, N., "Broadband achromatic dielectric metalenses," Light Sci. Appl. 7(1) (2018).

[31] Chen, W. T., Zhu, A. Y., Sanjeev, V., Khorasaninejad, M., Shi, Z., Lee, E. and Capasso, F., “A broadband achromatic metalens for focusing and imaging in the visible," Nat. Nanotechnol. 13(3), 220-226 (2018).

[32] Chen, W. T., Zhu, A. Y., Sisler, J., Bharwani, Z. and Capasso, F., "A broadband achromatic polarizationinsensitive metalens consisting of anisotropic nanostructures," Nat. Commun. 10(1), 1-7 (2019). 
[33] Byrnes, S. J., Lenef, A., Aieta, F. and Capasso, F., "Designing large, high-efficiency, high-numerical-aperture, transmissive meta-lenses for visible light," Opt. Express 24(5), 5110 (2016).

[34] She, A., Zhang, S., Shian, S., Clarke, D. R. and Capasso, F., "Large area metalenses: design, characterization, and mass manufacturing," Opt. Express 26(2), 1573 (2018).

[35] Kaplan, A. F., Xu, T., Wu, Y.-K. and Guo, L. J., "Multilayer pattern transfer for plasmonic color filter applications," J. Vac. Sci. Technol. B Microelectron. Nanom. Struct. (2010). 